\title{
An algebraic parameter estimation approach to the adaptive observer-controller based regulation of the boost converter
}

\author{
H. Sira-Ramírez* \\ CINVESTAV-IPN \\ Departamento de Ingeniería Eléctrica \\ Sección de Mecatrónica \\ Colonia San Pedro Zacatenco AP 14740 \\ 07300 México D.F., México \\ hsira@cinvestav.mx \\ Phone: 00-52-55-50613794
}

\author{
M. Spinetti-Rivera** \\ Universidad de Los Andes \\ Facultad de Ingeniería \\ Escuela de Ing. de Sistemas \\ Departamento de Sistemas de Control \\ Mérida 5101, Venezuela \\ marioseula.ve
}

\author{
E. Fossas-Colet**** \\ Instituto de Organización y Control \\ Av. Diagonal 647. Planta 11 \\ Edificio ETSEIB \\ Barcelona 08028, España \\ enric.fossasdupc.es \\ Phone: 0034-93-401-77-79
}

\begin{abstract}
In this article, a new approach is proposed for the problem of output voltage reference trajectory tracking in a boost converter with unknown, piecewise constant, load resistance values, under the restriction of noisy output voltage measurements alone.

We use a nonlinear algebraic parameter estimation approach for the fast computation of the actual value of the stepwise changing load. With this information, a reduced order nonlinear observer for the inductor current is updated as well as a static passive output feedback control law derived on the basis of the exact tracking error dynamics and Lyapunov stability considerations. The average nonlinear adaptive observer-controller scheme is implemented on the switch regulated converter using the averaging features of a $\Sigma-\Delta$ modulator.
\end{abstract}

\section{INTRODUCTION}

Most approaches to the control and regulation of DC to DC power converters, and other Power Electronics devices, rest on nonlinear adaptive feedback control techniques. An early contribution using feed-forward linear gains is that of Hiti and Borojevic [1]. Adaptive feedback controllers for power converters, from the context of passivity based control were contributed by Sira-Ramírez and his colleagues in [2] and from a backstepping adaptative control in [3]. The article by Sheng-Hua and Chang-Ming [4] propose the adaptive control of switching-mode rectifiers with robust voltage regulation control basing their developments on a linearized transfer

* This work was supported by CINVESTAV-IPN, by the Universidad de Castilla-La Mancha through a sabbatical year financed by the Spanish Ministry of Education and Science through the Secretaría de Estado de Universidades e Investigación and its Dirección General de Universidades

** Doctoral student at the Universidad Politécnica de Cataluña, work supported by the European Commission Alban scholarship program

***Work supported by the Spanish Ministry of Education and Science and Technology under project DPI2004-O68H-CO2-02 function and linear load adaptation. Adaptation within reference trajectory tracking problems is the main concern of Midya and co-workers [5] for buck and boost converters. Calvente et al. [6] propose the use of magnetic couplings to eliminate right half-plane zeros, i.e., non-minimum phase problems, in boost converters requiring adaptive feedback control. Finally, the work of Chattopadhyay and colleagues [7] present a digital implementation of a line current shaping algorithm for three phase high power factor boost rectifier without input voltage sensing.

In [8] a model reference adaptive control (MRAC) scheme is applied to a three-phase three-level neutral-point-clamped boost rectifier. This control strategy is developed with a view to regulate DC output and neutral point voltages and to reduce the influence of parameter variations while maintaining unity power factor. The proposed controller is based on the use of a feedback linearization technique followed by a robust MRAC scheme allowing the design of a suitable controller applied to the plant.Sanchis and his colleagues [9] treat the case of a Boost DC-AC inverter proposing an adaptive feedback control using a nested multi-loop strategy along with suitable low pass filters. A sliding mode control approach for uncertain dc to dc power converters is presented in Tan et al. [10]. To reduce switching frequency deviation in the events of line and load variations, an adaptive feed-forward control scheme that varies the hysteresis band according to the change of line input voltage and an adaptive feedback control scheme that varies the control parameter (i.e., sliding coefficient) according to the change of the output load are proposed.

More recently, Xu et al. [15] present a notch filter approach based in the frequency domain phase plot using a rather simple PI control scheme and a classical lag-lead 
controller. The works of Ben-Yaakov and Linekin [11] use adaptive control schemes for a high voltage converter in a piezoelectric transformer undergoing severe load variations which is based on the concept of maximum power point frequency for the maximum of power tracking. In [12] a PID implementation of and adaptive Pulse-width-Modulation based sliding mode controller is developed for the Boost converter. A rather efficient PI, state based, adaptive control scheme is presented in the work of Ratanapanachote et al. in [14]. A digital control algorithm capable of separately specifying the desired output voltage and transient response for a synchronous buck converter, operating in voltage mode, was developed by Oliva and his coworkers in [13]. A zero steady-state error in the output voltage is obtained with the aid of additional dynamics to allow the controller to track a load change and update the reference to a new load state. The specifications of the control algorithm are achieved by pole placement using complete state feedback.

In this article, we propose the suitable combination of adaptive static exact tracking error dynamics passive output feedback controllers, which in this case is indeed an observed state based feedback controller, along with an adaptive, reduced order nonlinear observer for the output voltage regulation, and output voltage reference trajectory tracking, of a boost converter with unknown piece-wise constant load parameter values.

Section 2 deals with a detailed description of the certainty equivalence observer and controller with proofs of their exponentially asymptotic features. Section 2 also describes the non-asymptotic, algebraic, on-line load parameter estimator. Section 3 is devoted to described the simulated performance of the proposed observer-controller-identifier scheme. Section 4 presents the conclusions and suggestions for further work in this area.

\section{ASSUMPTIONS PROBLEM FORMULATION AND MAIN RESULTS}

Consider the following boost converter model

$$
\begin{aligned}
L \dot{i} & =-u v+E \\
C \dot{v} & =u i-\frac{v}{R} \\
y & =v
\end{aligned}
$$

where $x_{1}$ is the inductor current $i, x_{2}$ is the output voltage $v$, supplied to the load. $R$ is the unknown resistor subject to unforseen step changes of varying and unknown amplitudes.

The normalization of the model (2.2) is carried out by means of the following, load independent, state and time coordinates transformation:

$$
x_{1}=\frac{1}{E} \sqrt{\frac{L}{C}} i, \quad x_{2}=\frac{v}{E}, \quad \tau=t \sqrt{L C}
$$

The normalized model is found to be

$$
\begin{aligned}
& \frac{d x_{1}}{d \tau}=-u x_{2}+1 \\
& \frac{d x_{2}}{d \tau}=u x_{1}-\frac{x_{2}}{Q}
\end{aligned}
$$

where $Q=\frac{1}{R} \sqrt{\frac{L}{C}}$ is the quality factor of the normalized circuit, input $u$ is average $[0,1]$

We assume that only the output voltage $y$ is available for measurement.

\section{A. Problem Formulation}

Given a normalized average model of the switched mode power supply of the boost type (2.2), with unknown resistive normalized load $Q$, subject to sudden step-like unknown changes, devise, on the basis of the measured output $y$ alone, an output feedback control scheme which robustly drives the output voltage to accurately track a given smooth output voltage reference trajectory $y^{*}(\tau)$.

\section{B. A certainty equivalence observer-controller}

Our strategy is to first derive a reduced order observer tracking error dynamics passive output feedback control scheme as if the unknown load were perfectly known. We then propose an on-line, non-asymptotic, fast load parameter estimator of the algebraic type which requires knowledge of the converter state variables and, for the feed-forward part, knowledge of the load. The observer controller scheme for uncertain plant is completed by feeding the actual fast computed load parameter into both the observer and the feedforward part of the controller. The required inductor current for the controller will be obtained from the adapted observer.

In the following two sections we derive the reduced observer static passivity based controller scheme as if the unknown load parameter were completely known. We also use the normalized average plant equations (2.2) in our developments.

\section{A reduced order average nonlinear observer for the inductor current}

Consider the average inductor current dynamics equation for the normalized plant

$$
\frac{d x_{1}}{d \tau}=-u y+1
$$

A time-varying, input dependent, nonlinear reduced order observer of the form:

$$
\frac{d \hat{x}_{1}}{d \tau}=-u y+1+\lambda u\left(x_{1}-\hat{x}_{1}\right)
$$

leads to the following estimation error equation, with $e_{1 o}=$ $x_{1}-\hat{x}_{1}$ :

$$
\dot{e}_{1 o}=-\lambda u e_{1 o}
$$

Setting $\lambda$ to be a sufficiently large positive constant and since the average control input $u$ is strictly bounded within the interval $[0,1]$ then clearly, $e_{1} \rightarrow 0$ in an exponentially asymptotically stable fashion.

Rewriting the proposed observer as

$$
\frac{d \hat{x}_{1}}{d \tau}=-u y+1+\lambda\left(u x_{1}-u \hat{x}_{1}\right)
$$

and since $u$ is assumed to be a known quantity then $u \hat{x}_{1}=$ $\widehat{u x_{1}}$. This means that the second dynamic equation of the 
plant (2.4) actually represents an implicit measurement of the required product signal $u x_{1}$ specified in terms of the output time derivative. Using this equation we obtain:

$$
u x_{1}=\frac{d y}{d \tau}+\frac{y}{Q}
$$

Substituting this last expression in the proposed observer (2.8), and defining an auxiliary variable $\zeta$ to be given by $\zeta=\hat{x}_{1}+\lambda y$, we obtain the following expression for the reduced order average input current observer

$$
\begin{aligned}
\dot{\zeta} & =-\left(1+\lambda^{2}\right) u y+1+\lambda\left(\frac{y}{Q}-u z\right) \\
\hat{x}_{1} & =\zeta-\lambda y
\end{aligned}
$$

Note that the proposed reduced order observer depends explicitly on the normalized load parameter $Q$. We shall be using the above observer as a certainty equivalence observer in which the parameter $Q$ will be replaced by an on-line algebraic estimate of the unknown load. This estimate will be denoted by $Q_{e}$.

\section{An exact tracking error dynamics passive output based linear time-varying feedback controller}

Suppose that in correspondence with a given output voltage reference trajectory $y^{*}(\tau)=x_{2}^{*}(\tau)$, we can compute a set of nominal average reference state and average input signals: $x_{1}^{*}(\tau), u^{*}(\tau)$, such that the nominal system is uniformly valid:

$$
\begin{aligned}
& \frac{d x_{1}^{*}(\tau)}{d \tau}=-u^{*}(\tau) x_{2}^{*}(\tau)+1 \\
& \frac{d x_{2}^{*}(\tau)}{d \tau}=u^{*}(\tau) x_{1}^{*}(\tau)-\frac{x_{2}^{*}(\tau)}{Q}
\end{aligned}
$$

The exact tracking error dynamics, characterized by $e_{1}=$ $x_{1}-x_{1}^{*}(\tau), e_{2}=x_{2}-x_{2}^{*}(\tau)$, is governed by:

$$
\begin{aligned}
& \frac{d e_{1}}{d \tau}=-u(\tau) e_{2}(\tau)-x_{2}^{*}(\tau) e_{u} \\
& \frac{d e_{2}}{d \tau}=u e_{1}-\frac{e_{2}}{Q}+x_{1}^{*}(\tau) e_{u}
\end{aligned}
$$

where $e_{u}=u-u^{*}(\tau)$.

The passive output corresponding to the exact tracking error dynamics (2.12) is given by

$$
e_{z}=-x_{2}^{*}(\tau) e_{1}+x_{1}^{*}(\tau) e_{2}
$$

Indeed, considering the total tracking error stored energy $H_{e}=0.5\left(e_{1}^{2}+e_{2}^{2}\right)$, we have:

$$
\dot{H}_{e}=\left[-x_{2}^{*}(\tau) e_{1}+x_{1}^{*}(\tau) e_{2}\right] e_{u}-\frac{e_{2}^{2}}{Q} \leq e_{z} e_{u}
$$

Clearly, for any strictly positive constant parameter $\gamma$, the following linear, time-varying, passive output feedback controller makes the origin of the tracking error space, $e=\left(e_{1}, e_{2}\right)$, into an exponentially asymptotically stable equilibrium point,

$$
\begin{aligned}
e_{u} & =-\gamma e_{z}=-\gamma\left[-x_{2}^{*}(\tau) e_{1}+x_{1}^{*}(\tau) e_{2}\right] \\
& =-\gamma\left[-x_{2}^{*}(\tau) x_{1}+x_{1}^{*}(\tau) x_{2}\right]
\end{aligned}
$$

which is derived, directly, from consideration of the positive definite Lyapunov function candidate $H_{e}$ defined above.

The exact tracking error dynamics passive output feedback (ETEDPOF) control law is rewritten as follows:

$$
u=u^{*}(\tau)-\gamma\left[-x_{2}^{*}(\tau) x_{1}+x_{1}^{*}(\tau) x_{2}\right]
$$

Clearly, this feedback control law requires the entire average state vector components: $\left(x_{1}, x_{2}\right)=\left(x_{1}, y\right)$, for its implementation. We propose the following output feedback tracking controller:

$$
u=u^{*}(\tau)-\gamma\left[-x_{2}^{*}(\tau) \hat{x}_{1}+x_{1}^{*}(\tau) y\right]
$$

with $\hat{x}_{1}$ being the estimate of the average input current, given by the nonlinear reduced order observer (2.10).

\section{E. A non-asymptotic algebraic load parameter estimator}

Consider the normalized output voltage dynamics with $x_{1}$ replaced by its asymptotic estimate $\hat{x}_{1}$, which evidently, is at our disposal:

$$
\frac{d y}{d \tau}=u(\tau) \hat{x}_{1}(\tau)-\frac{y}{Q}
$$

Multiplying out by the normalized time $\tau$ and integrating by parts the resulting left hand side, yields, after equating to the corresponding integration in the right hand side of (2.18):

$$
\begin{aligned}
\int_{0}^{\tau} \tau \frac{d y}{d \tau} d \tau & =\tau y-\int_{0}^{\tau} y(\tau) d \tau \\
& =\int_{0}^{\tau} \tau u(\tau) \hat{x}_{1}(\tau) d \tau-\frac{1}{Q} \int_{0}^{\tau} \tau y(\tau) d \tau
\end{aligned}
$$

Solving for $1 / Q$ one obtains:

$$
\begin{aligned}
\frac{1}{Q} & =\frac{-\tau y+\int_{0}^{\tau} y(\tau) d \tau+\int_{0}^{\tau} \tau u(\tau) \hat{x}_{1}(\tau) d \tau}{\int_{0}^{\tau} \tau y(\tau) d \tau} \\
& =\frac{-\tau y+\int_{0}^{\tau}\left[y(\tau)+\tau u(\tau) \hat{x}_{1}(\tau)\right] d \tau}{\int_{0}^{\tau} \tau y(\tau) d \tau}
\end{aligned}
$$

We address this computation formula for $1 / Q$ as the algebraic estimator for the unknown load.

The above formula for $Q$ is singular at time $t=0$, i.e., it is undefined as it yields a quotient of the form: $0 / 0$. After $\Delta>0$ units of time have elapsed, the above quotient (2.20) is well defined. Clearly, neither the numerator nor the denominator may be identically zero, since then either $y$ or $\hat{x}_{1}$ satisfy differential equations which are independent of $Q$. This would be a contradiction. During the interval of time $[0, \Delta)$ we shall adopt an arbitrary constant value for the estimate of $1 / Q$. Since $Q$ will be changing, we adopt a resetting policy for the algebraic estimator. At the resetting times, $\left\{\tau_{r}, \tau_{r+1}, \ldots\right\}$, we recompute the estimate of $1 / Q$ using the shifted version of (2.20). We recall that the accurate computation takes $\Delta$ seconds to be performed. We thus propose an estimator for the normalized load parameter, 
$1 / Q$, as follows:

$$
\frac{1}{Q_{e s t}}=\left\{\begin{array}{l}
\text { arbitrary } \\
\text { for } \tau \in\left[\tau_{r}, \tau_{r}+\Delta\right) \\
\frac{-\left(\tau-\tau_{r}\right) y+\int_{\tau_{r}}^{\tau}\left[y(\tau)+\left(\tau-\tau_{r}\right) u(\tau) \hat{x}_{1}(\tau)\right] d \tau}{\int_{\tau_{r}}^{\tau}\left(\tau-\tau_{r}\right) y(\tau) d \tau} \\
\text { for } \tau \in\left[\tau_{r}+\Delta, \tau_{r+1}\right)
\end{array}\right.
$$

\section{F. Invariant filtering}

The measured signal $y$ in the preceding observer-controller scheme based on algebraic parameter estimations is never devoid of measurement noise. For this reason it is to be expected that the parameter estimation will be sensitive to such additive noises. In order to enhance the signal to noise ratio in the numerator and the denominator expression of the algebraic parameter identifier, we propose to carry out an independent filtering of the numerator and denominator signals but using two identical filters. Such an enhancement results in an accurate estimate of the load parameter $Q$. In the following expression we abusively combine the (normalized) time domain expressions with frequency domain expressions in order to emphasize the invariance of the proposed filtering operation.

$$
\frac{1}{Q_{\text {est }}}=\frac{\frac{1}{s^{2}}\left[-\tau y+\int_{0}^{\tau}\left[y(\tau)+\tau u(\tau) \hat{x}_{1}(\tau)\right] d \tau\right]}{\frac{1}{s^{2}}\left[\int_{0}^{\tau} \tau y(\tau) d \tau\right]}
$$

In summary, when the calculation resetting are used for the estimation of piecewise constant loads, we have the following expression for the algebraic estimator with second order integration invariant filtering:

$$
\frac{1}{Q_{e s t}}=\left\{\begin{array}{l}
\text { arbitrary } \\
\text { for } \tau \in\left[\tau_{r}, \tau_{r}+\Delta\right) \\
\frac{\frac{1}{s^{2}}\left[-\left(\tau-\tau_{r}\right) y+\int_{\tau_{r}}^{\tau}\left[y(\tau)+\left(\tau-\tau_{r}\right) u(\tau) \hat{x}_{1}(\tau)\right] d \tau\right]}{\frac{1}{s^{2}}\left[\int_{\tau_{r}}^{\tau}\left(\tau-\tau_{r}\right) y(\tau) d \tau\right]} \\
\text { for } \tau \in\left[\tau_{r}+\Delta, \tau_{r+1}\right)
\end{array}\right.
$$

\section{G. Sigma-Delta Modulation Implementation}

The previous results point to the average adaptive output feedback controller design. The obtained control input signal is to be interpreted as an average control input. The boost system is, nevertheless, commanded by a switch position function taking values in the discrete set $\{0,1\}$. Hence, a continuous to binary conversion is required for the designed feedback input signal that respects the properties of the average overall closed loop behavior of the system. We propose as a realization of the switched actuator the well known, $\Sigma-\Delta$, modulator option (addressed here as: "SigmaDelta" modulation, see the article by Sira-Ramírez and Silva Ortigoza, [16]). Other options are Pulse-Width-Modulation (PWM) schemes based on the interpretation of the bounded input signal as a duty ratio for the switched PWM policy to be implemented.

Given the average input signal $u_{a v}$ a continuous to binary valued conversion which leaves the average properties of the controlled system is given by:

$$
\begin{aligned}
u & =\frac{1}{2}(1+\operatorname{sign} z) \\
\frac{d z}{d t} & =u_{a v}-u
\end{aligned}
$$

It is not difficult to show that a sliding regime globally exists on the sliding surface $z=0$, achievable in finite time. We have the following result.

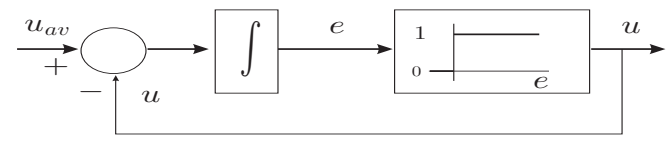

Fig. 1. $\quad \Sigma-\Delta$-modulator

Theorem 2.1: Consider the $\Sigma-\Delta$-modulator of Figure 1. Given a sufficiently smooth, bounded, signal $u_{a v}(t)$, then the integral error signal, $e(t)$, converges to zero in a finite time, $t_{h}$, and, moreover, from any arbitrary initial value, $e\left(t_{0}\right)$, a sliding motion exists on the perfect encoding condition surface, represented by $e=0$, for all $t>t_{h}$, provided the following encoding condition is satisfied for all $t$,

Proof.

$$
0<u_{a v}(t)<1
$$

The variables in the $\Sigma-\Delta$-modulator satisfy the following relations:

$$
\begin{aligned}
\dot{e} & =u_{a v}(t)-u \\
u & =\frac{1}{2}[1+\operatorname{sign}(e)]
\end{aligned}
$$

The quantity $e \dot{e}$ is given by:

$$
\begin{aligned}
e \dot{e} & =e\left[u_{a v}-\frac{1}{2}(1+\operatorname{sign}(e))\right] \\
& =-|e|\left[\frac{1}{2}(1+\operatorname{sign}(e))-u_{a v} \operatorname{sign}(e)\right]
\end{aligned}
$$

For $e>0$ we have $e \dot{e}=-e\left(1-u_{a v}\right)$, which, according with the assumption in (2.25) leads to $e \dot{e}<0$. On the other hand, when $e<0$, we have $e \dot{e}=-|e| u_{a v}<0$.

A sliding regime exists then on $e=0$ for all time $t$ after the hitting time $t_{h}$. Under ideal sliding, or encoding, conditions, $e=0, \dot{e}=0$, we have that the, so called, equivalent value of the switched output signal, $u$, denoted by $u_{e q}(t)$ satisfies:

$$
u_{e q}(t)=u_{a v}(t)
$$


An estimate of the hitting time $t_{h}$ is obtained by examining the modulator system equations with the worst possible bound for the input signal $u_{a v}$ in each of the two conditions: $e>0$ and $e<0$, along with the corresponding value of $u$.

Consider then that $e(0)>0$ at time $t=0$. We have for all $0<t \leq t_{h}^{+}$

$$
\begin{aligned}
e(t) & =e(0)+\int_{0}^{t}\left(u_{a v}(\sigma)-u(\sigma)\right) d \sigma \\
& =e(0)+\int_{0}^{t}\left(u_{a v}(\sigma)-1\right) d \sigma
\end{aligned}
$$

Since $e\left(t_{h}^{+}\right)=0$, we have:

$$
\begin{aligned}
0= & e(0)+\int_{0}^{t_{h}^{+}}\left(u_{a v}(\sigma)-1\right) d \sigma \leq e(0) \\
& +t_{h}^{+}\left[\sup _{t \in\left[0, t_{h}^{+}\right]} u_{a v}(t)-1\right] \\
& \leq e(0)+t_{h}^{+}\left[\sup _{t} u_{a v}(t)-1\right]
\end{aligned}
$$

Hence,

$$
t_{h}^{+} \leq \frac{e(0)}{1-\sup _{t} u_{a v}(t)}
$$

When $e(0)<0$ We have for all $0<t \leq t_{h}^{-}$,

$$
\begin{aligned}
e(t) & =e(0)+\int_{0}^{t}\left(u_{a v}(\sigma)-u(\sigma)\right) d \sigma \\
& =e(0)+\int_{0}^{t}\left(u_{a v} \sigma\right) d \sigma
\end{aligned}
$$

Since, as before, $e\left(t_{h}^{-}\right)=0$, we have:

$$
\begin{aligned}
0= & e(0)+\int_{0}^{t_{h}^{-}} u_{a v}(\sigma) d \sigma \geq e(0) \\
& +t_{h}^{-}\left[\inf _{t \in\left[0, t_{h}^{-}\right]} u_{a v}(t)\right] \\
& \geq e(0)+t_{h}^{-}\left[\sup _{t} u_{a v}(t)\right]
\end{aligned}
$$

Hence,

$$
t_{h}^{-} \leq \frac{-e(0)}{\inf _{t} u_{a v}(t)}=\frac{|e(0)|}{\inf _{t} u_{a v}(t)}
$$

The average $\Sigma-\Delta$-modulator output $u_{e q}$, ideally yields the modulator's input signal $u_{a v}(t)$ in an equivalent control sense.

\section{H. Main Result}

A certainty equivalence ETEDPOF controller, with the unmeasured state being supplied by a certainty equivalence asymptotically exponentially convergent, nonlinear, observer along with an algebraic, on-line, load parameter observer lie at the basis of our proposed combined feedback control scheme for the stabilization and trajectory tracking of uncertain dc to dc power converters undergoing severe load changes. Figure 2 depicts the proposed control scheme in a block diagram form.

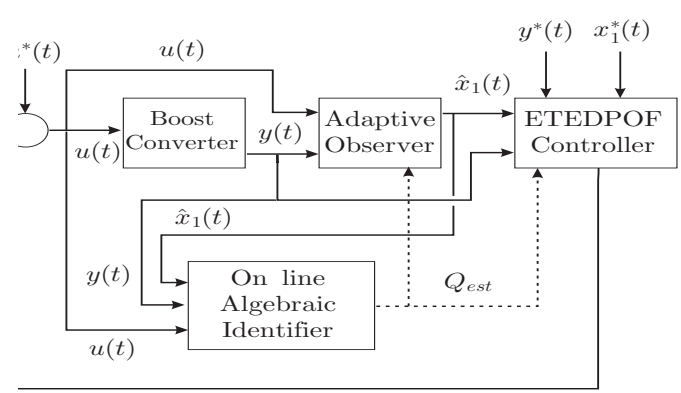

Fig. 2.

\section{Simulation Results}

We simulated the feedback controlled boost converter with the following changing values of the normalized parameter $Q$

$$
Q= \begin{cases}1.75 & \text { for } t \in[0,7.6) \\ 0.87 & \text { for } t \in[7.6,15.9) \\ 3.5 & \text { for } t \in[15.9, \infty)\end{cases}
$$

The control objective was set as one of output voltage regulation to a pre-specified normalized equilibrium value: $V_{d}=1.5$ Volt. The certainty equivalence version of the ETEDPOF controller (2.16) was specified as follows:

$$
u=\frac{1}{V_{d}}-\gamma\left(-V_{d} \hat{x}_{1}+\frac{V_{d}^{2}}{Q_{e s t}} y\right)
$$

where $Q_{\text {est }}$ stands for the algebraically estimated value of the piecewise constant, unknown, parameter $Q$. We have used: $y^{*}(\tau)=V_{d}, x_{1}^{*}(\tau)=V_{d}^{2} / Q_{\text {est }}$, and $u^{*}(\tau)=1 / V_{d}$. The parameter $\gamma$ was set to be $\gamma=1$.

The certainty equivalence observer for the normalized inductor current is given by:

$$
\begin{aligned}
\hat{x}_{1} & =\zeta-\lambda y \\
\dot{\zeta} & =-u y\left(1+\lambda^{2}\right)+1+\lambda\left(\frac{y}{Q_{e s t}}-u \zeta\right)
\end{aligned}
$$

In the simulations we have used a noisy measurement of the output voltage $y(t)=x_{2}+\xi(t)$ where $\xi(t)$ is a computer generated stochastic process, generated on the basis of zero mean piecewise constant random variable uniformly distributed in the interval $[-0.05,0.05]$. The normalized constant source voltage of nominal value 1 , was also set to be noisy and of the form: $1+\xi(t)$. The algebraic parameter estimator was reset on the basis of significant deviations of the output voltage from the desired constant reference equilibrium trajectory. The invariant filtering associated with the algebraic estimator was provided by means of two independent pure integrations on the numerator and denominator signals.

Figure 3 depicts the closed loop performance of the adaptive observer-controller scheme, feeder with the obtained estimations coming from the on-line, non-asymptotic, algebraic parameter estimator. The output voltage trajectory depicts the recovery features imposed by the output feedback control scheme under the severe load variations. The estimated normalized input current is shown along with the actual normalized inductor current. The performance of the 

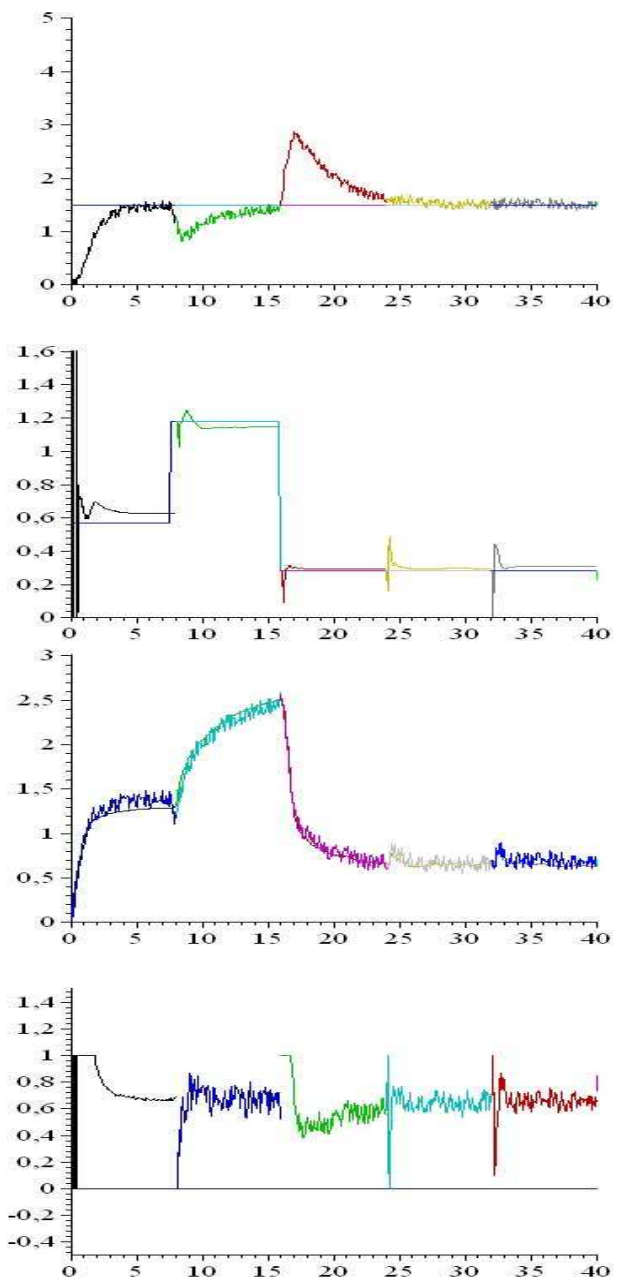

Fig. 3. Closed loop performance for average normalized Boost converter with adaptive reduced order nonlinear observer and adaptive ETEDPOF controller feed by an on-line algebraic parameter estimator

algebraic estimator generating $Q_{e s t}$ is also depicted in the simulations.

\section{CONCLUSIONS}

In this article, we have proposed an average output feedback controller for a rest-to-rest trajectory tracking task in an uncertain Boost converter with unknown, piece-wise constant, load parameter. The observed state feedback controller is an ETEDPOF feedback controller which requires the estimation of the input inductor current. The estimation of the inductor current is accomplished by means of a reduced order nonlinear, input-dependent, observer whose defining state is globally exponentially asymptotically stable to the inductor current unmeasured trajectory. Both, the static feedback controller and the dynamic input current observer are of the uncertainty equivalence type since they both depend on the unknown load resistor changing value. The load is on-line computed, in a non asymptotic fashion, using an fast algebraic parameter estimator and a resetting policy triggered by the deviations of the desired equilibrium value. The performance of the closed loop system was satisfactorily tested using rather noisy measurements of the output voltage variable.

Implementation of the proposed feedback controller in a laboratory environment requires a quite fast data acquisition card since the controller-observer-algebraic estimator scheme relies on fast digital computations. This task is being considered in detail at the present moment in connection with the boost and some other DC-to-DC Power converters.

\section{REFERENCES}

[1] Hiti, S.; Borojevic, D.; "Robust nonlinear control for boost converter". IEEE Transactions on Power Electronics, Vol. 10, No. 6, pp. 651-658, 1995.

[2] Sira-Ramrez, H. Ortega R. and Garca-Esteban M., "Adaptive Passivity Based Control of Average DC-to-DC Power Converter Models" International Journal of Adaptive Control and Signal Processing Vol. 12, pp. 63-80, 1998.

[3] Sira-Ramrez, H., Rios-Bolvar, M., and Zinober, A.S.I. "Adaptive Dynamical Input-Output Linearization of DC to DC Power Converters: A Backstepping Approach" International Journal of Robust and Nonlinear Control, Vol. 7, No. 3, pp. 279-296, 1997.

[4] Sheng-Hua Li, Chang-Ming Liaw, "Paralleled DSP-based soft switching mode rectifiers with robust voltage regulation control". IEEE Transac tions on Power Electronics, Vol. 19, No. 4, pp. 937-946, 2004.

[5] Midya, P., Haddad, K., Miller, M., Buck or boost tracking power converter. IEEE Power Electronics Letters, Vol. 2, No. 4, pp. 131-134, 2004

[6] Calvente, J., Martinez-Salamero, L., Valderrama, H., Vidal-Idiarte, E., Using magnetic coupling to eliminate right half-plane zeros in boost converters. IEEE Power Electronics Letters, Volume 2, No. 2, pp. 5862, 2004.

[7] Chattopadhyay, S., Ramanarayanan, V.,, "Digital implementation of a line current shaping algorithm for three phase high power factor boost rectifier without input voltage sensing". IEEE Transactions on Power Electronics, Vol. 19, No. 3, pp. 709 - 721, 2004

[8] Yacoubi, L., Al-Haddad, K., Dessaint, L.A., and Fnaiech, F. "A DSPbased implementation of a nonlinear model reference adaptive control for a three-phase three-level NPC boost rectifier prototype". IEEE Transactions on Power Electronics, Volume 20, No. 5, pp. 1084-1092, 2005.

[9] Sanchis, P., Ursaea, A., Gubia, E., Marroyo, L., "Boost DC-AC inverter: a new control strategy". IEEE Transactions on Power Electronics, Vol. 20, No. 2, pp. 343-353, 2005

[10] Tan, S.-C., Lai, Y.M., Tse, C.K., “ Implementation of Pulse-widthModulation Based Sliding Mode Controller for Boost Converters". IEEE Power Electronics Letters, Vol. 3, No. 4, pp. 130-135, 2005.

[11] Ben-Yaakov S., and Lineykin, S. " Maximum Power Tracking of Piezoelectric Transformer HV Converters Under Load Variations". IEEE Transactions on Power Electronics, Vol. 21, No. 1, pp. 73-78, 2006

[12] Tan, S.C., Lai, Y.M., Tse, C.K., Cheung, M.K.H., "Adaptive Feedforward and Feedback Control Schemes for Sliding Mode Controlled Power Converters". IEEE Transactions on Power Electronics, Vol. 21, No. 1, pp. 182-192, 2006.

[13] Oliva, A.R., Ang, S.S., Bortolotto, G.E., "Digital Control of a VoltageMode Synchronous Buck Converter" IEEE Transactions on Power Electronics, Vol. 21, No. 1, pp. 157-163, 2006.

[14] Ratanapanachote, S., Cha, H.J., Enjeti, P.N., "A Digitally Controlled Switch Mode Power Supply Based on Matrix Converter". IEEE Transactions on Power Electronics, Vol. 21, No. 1, pp. 124-130, 2006.

[15] Xu, X., Khambadkone, A.M., Leong, T.M., Oruganti, R., “A 1-MHz Zero-Voltage-Switching Asymmetrical Half-Bridge DC/DC Converter: Analysis and Design". IEEE Transactions on Power Electronics, Volume 21, No. 1, pp. 105-113, 2006.

[16] H. Sira-Ramírez and R. Silva-Ortigoza, " Sliding Mode, Sigma-Delta Modulation Control of the Boost Converter, Asian Journal of Control Vol. 7, No. 4, pp. 1-7, December 2005 